\title{
A dimensão espacial e temporal da diversidade de peixes da zona litoral vegetada de lagoas marginais da planície de inundação do rio Cuiabá, Pantanal, Brasil
}

\author{
Leandro Junior Baginski ${ }^{1,3}$, Alexandro Cezar Florentino ${ }^{1}$, Izaias Médice Fernandes ${ }^{1}$, \\ Jerry Magno Ferreira Penha ${ }^{2} \&$ Lúcia Aparecida de Fátima Mateus ${ }^{2}$
}

Biota Neotropica v7 (n3) - http://www.biotaneotropica.org.br/v7n3/pt/abstract?article+bn04007032007

\author{
Recebido em 17/06/07 \\ Versão Reformulada recebida em 14/08/07 \\ Publicado em 09/11/07 \\ ${ }^{1}$ Programa de Pós-graduação em Ecologia e Conservação da Biodiversidade, \\ Universidade Federal de Mato Grosso - UFMT, CEP 78060-900, Cuiabá, MT, Brasil \\ e-mail: alexandrocezar@yahoo.com.br,biomedice@hotmail.com \\ ${ }^{2}$ Núcleo de Estudos Ecológicos do Pantanal - NEPA, Instituto de Biociências, \\ Universidade Federal de Mato Grosso, CEP 78060-900, Cuiabá, MT, Brasil \\ e-mail:jpenha@ufmt.br,lmateus@ufmt.br \\ ${ }^{3}$ Autor para correspondência: Leandro Junior Baginski,e-mail: leandrobaginski@yahoo.com.br
}

\begin{abstract}
Baginski, L.J., Florentino, A.C., Fernandes, I.M., Penha, J.M.P. \& Mateus, L.A.F. The spatial and temporal dimension of fish diversity of the vegetated littoral zone of marginal lagoons of the Cuiabá river floodplain, Pantanal, Brazil. Biota Neotrop. Sep/Dez 2007 vol. 7, no. 3 http://www.biotaneotropica.org.br/v7n3/pt/ abstract?article+bn04007032007. ISSN 1676-0603.

The present work has evaluated the spatial and temporal distribution of fish species richness of the vegetated littoral zone of 15 marginal lagoons in the floodplain of the Cuiabá River, Pantanal. The lagoons were sampled in three periods along of the dry season (June, September and December of 2005), through nine sieve throws distributed systematically at a minimum distance of five meters from each other. We caught 12,784 individuals belonging to seven orders, 21 families and 98 species of fish. Most of the captured species were rare and presented restricted geographical distribution. Drought intensification did not affect the spatial species distribution and the loss of species as dry season proceeded was proportional in the different orders. The total abundance of individuals as well as the richness varied with time. Rarefaction curves indicated that the reduction in richness was an artifact of the reduction in the abundance of individuals. When we compared the estimated richness under a same abundance level we did not find difference among the richness of species in the beginning and at the end of the dry season. Richness estimators indicated that to distribute the samples in the time was an appropriate strategy to reduce the problem of the false absences and to improve the efficiency of the sampling. Thus, our results suggest that for the design of reserves in the Pantanal, areas with a large number of lagoons of different forms and sizes would conserve the regional fish diversity, as the distribution of most small size species is restricted to a few lagoons.

Keywords: effect of dry season, abundance distribution, spatial distribution, local extinction.
\end{abstract}

Resumo

Baginski, L.J., Florentino, A.C., Fernandes, I.M., Penha, J.M.P. \& Mateus, L.A.F. A dimensão espacial e temporal da diversidade de peixes da zona litoral vegetada de lagoas marginais da planície de inundação do rio Cuiabá, Pantanal, Brasil. Biota Neotrop. Sep/Dez 2007 vol. 7, no. 3 http://www.biotaneotropica.org.br/ v7n3/pt/abstract?article+bn04007032007. ISSN 1676-0603.

O presente trabalho avaliou a distribuição espacial e temporal da riqueza de espécies de peixes da zona litoral vegetada de 15 lagoas marginais da planície de inundação do Rio Cuiabá, Pantanal. As lagoas foram amostradas em três períodos ao longo da sessão seca (junho, setembro e dezembro de 2005), por meio de nove lances de peneira, distribuídos sistematicamente a uma distância mínima de cinco metros um do outro. Capturamos 12.784 indivíduos pertencentes a sete ordens, 21 famílias e 98 espécies. A maioria das espécies capturadas foi rara e apresentou distribuição geográfica restrita. A intensificação da seca não afetou a distribuição espacial das espécies e a perda de espécies nas diferentes ordens, ao longo da seca, foi proporcional. Tanto a abundância total de indivíduos quanto a riqueza variou no tempo. As curvas de rarefação indicaram que a redução na riqueza foi um artefato da redução na abundância de indivíduos. Quando comparamos as riquezas estimadas sob um mesmo nível de abundância não encontramos diferença entre a riqueza de espécies no início e no final da seca. Os estimadores de riqueza de espécies indicaram que distribuir as amostras no tempo foi uma estratégia adequada para reduzir o problema das falsas ausências e melhorar a eficiência da amostragem. Assim, nossos resultados sugerem que para o delineamento de reservas no Pantanal, áreas com um grande número de lagoas com diferentes formas e tamanhos conservaria um maior contingente da diversidade regional de peixes, pois a maioria das espécies de pequeno porte apresenta distribuição restrita a poucas lagoas.

Palavras-chave: efeito da seca, distribuição de abundâncias, distribuição espacial, extinção local. 


\section{Introdução}

Uma meta comum em estudos de ecologia de comunidades é encontrar padrões de distribuição e abundância das espécies e determinar os fatores responsáveis pelos padrões (Begon et al. 2006). Em geral, estes fatores são somente subconjuntos dos possíveis determinantes da organização da comunidade (Hinch \& Collins 1991). Dentre os padrões mais abordados em ecologia de comunidades estão aqueles relacionados com a diversidade de espécies (número, identidade e as características das mesmas), bem como aqueles relativos às variações temporais nas abundâncias (Hoeinghaus et al. 2003, Miranda \& Mazzoni 2003, Webster 2003, Lugendo et al. 2007).

Mudanças em comunidades naturais ocorrem no espaço e no tempo. Por exemplo, locais com altas taxas de mudança temporal acumulam mais espécies em um determinado período de tempo que locais com mudanças restritas (Chalcraft et al. 2004). Um entendimento detalhado da diversidade pode ser alcançado através de estudos que particionam a diversidade ao longo das dimensões de diferentes habitats, com tamanhos de amostras muito maiores, permitindo assim, medir a diversidade em diferentes subconjuntos da comunidade (DeVries et al. 1997).

O Pantanal, uma das maiores áreas alagáveis do mundo, ainda carece de estudos que avaliem a diversidade a estrutura e a dinâmica das comunidades de peixes. Entretanto, destacam-se os trabalhos de Súarez et al. (2001, 2004) no Pantanal da Nhecolândia, que avaliaram os padrões de composição e diversidade nas comunidades de peixes em lagoas sazonalmente isoladas. Os autores sugerem que, algumas características ambientais (p. ex., profundidade da lagoa, cobertura de macrófitas, distância da lagoa ao rio e tempo de isolamento da lagoa) estão entre os principais determinantes da distribuição das espécies, e que a intensidade das cheias é de grande importância, por afetar a colonização das lagoas e sua diversidade.

Este trabalho teve como objetivo avaliar o efeito da variação espacial e temporal do ambiente, durante o período da seca, na riqueza de espécies e distribuição de abundâncias nas assembléias de peixes da zona litoral vegetada de lagoas marginais da planície de inundação do rio Cuiabá, assim como avaliar a eficiência amostral do delineamento utilizado.

\section{Material e Métodos}

\section{1. Área de estudo}

As coletas foram realizadas em lagoas da planície de inundação do rio Cuiabá, localizadas dentro da Reserva Particular do Patrimônio Natural SESC Pantanal (RPPN SESC Pantanal) e seu entorno, situada entre as coordenadas $16^{\circ} 30^{\prime}$ a $16^{\circ} 44^{\prime} \mathrm{S}$ e $56^{\circ} 20^{\prime}$ a $56^{\circ} 30^{\prime} \mathrm{W}$ (Figura 1).

O rio Cuiabá pertence à porção norte do Pantanal Matogrossense. $\mathrm{O}$ rio recebe esse nome após a confluência dos rios Manso e Cuiabazinho e corre em direção ao sul, até a desembocadura com o rio Paraguai. Como quase todos os rios da região, trata-se de um rio de meandros, que associado à sua baixa declividade, propicia inundações anuais (Hamilton et al. 1996), as quais geralmente ocorrem de dezembro a março. Ao longo de suas margens encontra-se um complexo de lagoas de diferentes tipos, formadas pelas depressões naturais ou por mudanças naturais do curso do rio.

\section{Amostragem}

Foram amostradas 15 lagoas, sendo oito localizadas no interior da RPPN-SESC Pantanal e sete no entorno da reserva. Em cada lagoa foram realizadas três coletas nos meses de junho, setembro e dezembro de 2005, período de águas baixas na região. As amostras foram

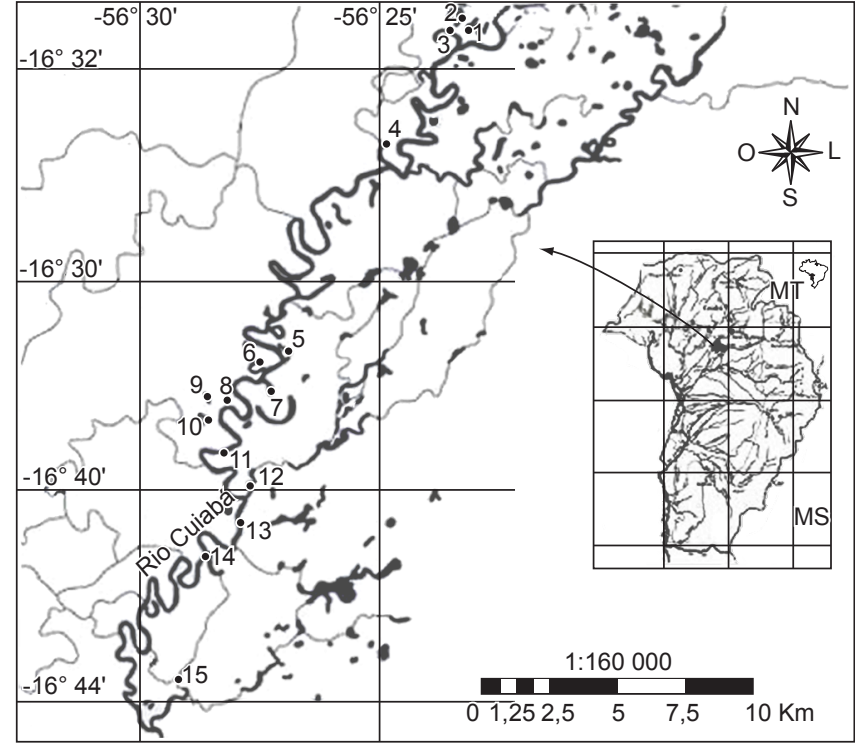

Figura 1. Localização das lagoas amostradas na bacia do rio Cuiabá, Pantanal, Brasil. As lagoas da margem esquerda do rio estão localizadas dentro da reserva RPPN SESC-Pantanal e as da margem direita fora da reserva.

Figure 1. Local of sampled lagoons on Cuiabá river basin, Pantanal, Brazil. The lagoons located on the left margin of the river are inside the RPPN SESC-Pantanal reserve and the ones located on the right margin are outside the reserve.

distribuídas no tempo, a fim de se avaliar as alterações na riqueza de espécies provocadas pela seca.

As amostras foram realizadas na zona litoral vegetada das lagoas, dominada por macrófitas flutuantes, especialmente Eichhornia azurea, Eichhornia crassipes e Salvinia sp.. Em cada lagoa foram aplicados nove lances de peneira, distribuídos sistematicamente a uma distância mínima de cinco metros um do outro. A peneira utilizada tinha um saco em forma de funil, com $1 \mathrm{~m}^{2}$ de boca e profundidade de $1 \mathrm{~m}$, confeccionada com tela de nylon de $1,5 \mathrm{~mm}$ de abertura de malha. Apesar da seletividade da peneira - ineficiente para as espécies de grande porte, esta é eficiente para a coleta de peixes de pequeno porte, que são as formas predominantes nas zonas litorais vegetadas.

Os peixes coletados foram fixados em formol $10 \%$ e preservados em álcool 70\%. Posteriormente, os mesmos foram identificados com auxílio de literatura especializada (Britski et al. 1999, Reis et al. 2003) e a confirmação feita por especialistas do Museu de Zoologia da USP (MZUSP). Exemplares testemunhos se encontram depositados no Laboratório de Ecologia e Manejo de Recursos Pesqueiros/UFMT.

\section{Análise de dados}

Com o objetivo de comparar a distribuição de abundância das espécies ("Whittaker plots") na comunidade de peixes, as abundâncias das espécies (n) foram transformadas utilizando $\log _{10}(n+1)$ (Magurran 2004). A distribuição de abundância das espécies foi plotada utilizando Log na base 2 e comparada com os modelos de distribuição das abundâncias de espécies (Distribuição Log normal e Série logarítimica) (Magurran 1988b). Os modelos foram ajustados utilizando o programa estatístico BIO-DAP (Magurran 1988a).

Para comparar a freqüência de ocorrência das espécies nas lagoas e a distribuição de frequiência de espécies nas diferentes ordens, entre o início (junho) e o final (dezembro) da sessão seca, foi utilizado o teste não paramétrico de Kolmogorov-Smirnov $\left(\mathrm{D}_{\max }\right)$. Em cada lagoa (unidades amostrais) foi obtido o número de espécies por ordem 
(variável dependente) que foi comparado em diferentes momentos no tempo (variável independente).

Para avaliar possíveis diferenças na riqueza de espécies entre o início e o final da sessão de seca foi utilizada a técnica de rarefação (Sanders 1968). Essa técnica estima a riqueza de espécies esperada para cada subamostra de indivíduos de uma dada amostra, e é especialmente útil para comparar amostras de tamanhos diferentes (Gotelli \& Graves 1996).

Com a finalidade de avaliar a eficiência de amostragem, comparamos o número de espécies registrados nas campanhas com o número de espécies esperado para a área, obtidos com os estimadores Chao 2 e Jackknife de segunda ordem, no programa EstimateS (Colwell 2005)

\section{Resultados}

Foram capturados 12.784 indivíduos pertencentes a sete ordens, 21 famílias e 98 espécies de peixes. A ordem com maior número de espécies foi Characiformes, com 53 espécies, seguida por Siluriformes (21 espécies), Perciformes (13 espécies), Gymnotiformes ( 7 espécies) e as demais somando quatro espécies.

A distribuição de abundâncias das espécies na comunidade mostrou que poucas espécies foram muito abundantes, algumas com abundâncias intermediárias e a maioria com baixa abundância (Figura 2), sendo que 73,5\% das espécies apresentaram abundância inferior a 100 indivíduos e 39,7\% das espécies foram representadas por 10 ou menos indivíduos. Por outro lado, a espécie mais abundante, Serrapinnus calliurrus representou 22,9\% da amostra (2.798 indivíduos). Apesar das abundâncias apresentarem distribuição caracteristicamente lognormal, estas foram igualmente bem descritos pela série logarítmica (lognormal, $\mathrm{x}^{2}=12,17 ; \mathrm{df}=9 ; \mathrm{p}>0,20$; série logarítimica, $\left.\mathrm{x}^{2}=15,53 ; \mathrm{gl}=11 ; \mathrm{p}>0,1\right)$ (Figura 3).

Embora algumas espécies (Gymnocorymbus ternetzi, Moenkhausia sanctaefilomenae, e Corydoras hastatus) sejam exploradas com fins ornamentais e outras (Synbranchus marmoratus e Gymnotus cf. carapo) utilizadas como iscas vivas, nenhuma das 98 espécies capturadas é citada na lista vermelha da The Word Conservation Union (IUCN) como espécie ameaçada de extinção.

Nos três períodos de coleta, os maiores indivíduos capturados, que não ultrapassaram $40 \mathrm{~cm}$ de comprimento total, pertenciam às ordens

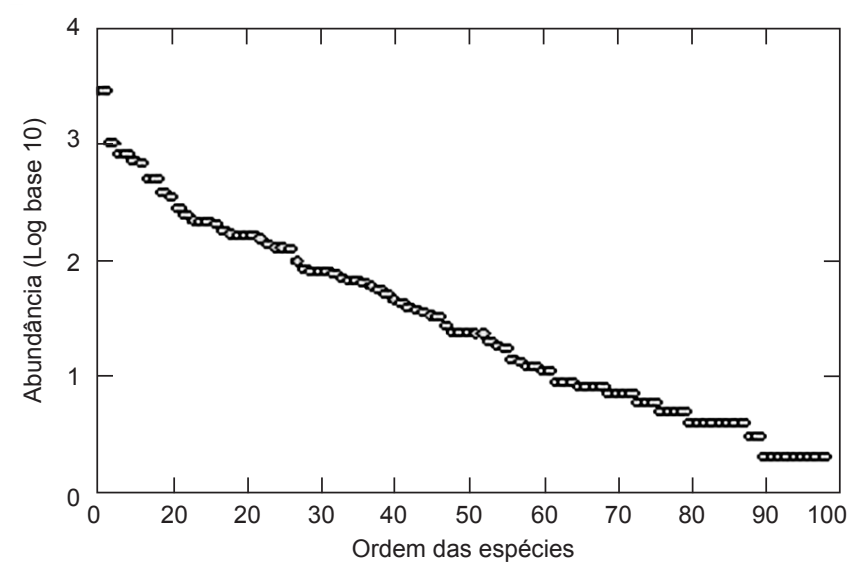

Figura 2. Distribuição de abundâncias da comunidade de peixes da zona litoral vegetada de 15 lagoas marginais do rio Cuiabá, Pantanal, Brasil. Os dados são a soma dos três períodos de amostragens (junho, setembro e dezembro de 2005).

Figure 2. Fish species rank of the vegetated littoral zone for 15 floodplain lagoons in the Cuiabá River Basin, Pantanal, Brazil. The data are the sum of the three periods sampled (June, September, and December of 2005).
Gymnotiformes e Synbrachiformes. Todos os demais indivíduos capturados apresentaram tamanho corporal pequeno, sendo principalmente espécies sedentárias ou migradoras de curta distância.

A maioria das espécies apresentou distribuição geográfica restrita e a intensificação do período de seca não afetou a distribuição das espécies. Em junho 52\% (42/80) das espécies tinham ocorrência restrita a quatro lagoas ( $25 \%$ do total amostrado). Em dezembro este percentual aumentou para 60\% (41/68). Entretanto, esta variação de $8 \%$ em relação ao início da seca (Figura 4) não foi estatisticamente significativa $\left(D_{\text {max }}=0,333\right.$ e $\left.\mathrm{p}=0,341\right)$.

A intensificação da seca não afetou a distribuição de freqüência de espécies nas diferentes ordens $\left(\mathrm{D}_{\max }=0,143 \mathrm{e} \mathrm{p}=1\right)$ (Figura 5). Entre as campanhas de junho e dezembro todas as ordens, à exceção de Lepidosireniformes e Synbranchiformes, perderam aproximadamente a mesma proporção de espécies.

Tanto a abundância total de indivíduos quanto a riqueza variaram no tempo (Tabela 1). No mês de junho foram coletadas $81,6 \%$ do total das espécies, no mês de setembro $75,5 \%$ e no de dezembro $69,4 \%$. Cerca de $50 \%$ das espécies foram comuns aos três períodos de coleta (Figura 6). Por outro lado, 22,4\% das espécies ocorreram em apenas um período de coleta, sendo que $10,2 \%$ foram coletadas somente no mês de junho, 7,1\% foram exclusivas ao mês de setembro e 5,1\% ao mês de dezembro (Tabela 1).

As curvas de rarefação indicaram que a redução na riqueza foi um artefato da redução na abundância. Quando comparamos as riquezas estimadas sob um mesmo nível de abundância não encontramos diferença na riqueza de espécies entre o início e o final da seca (Figura 7).

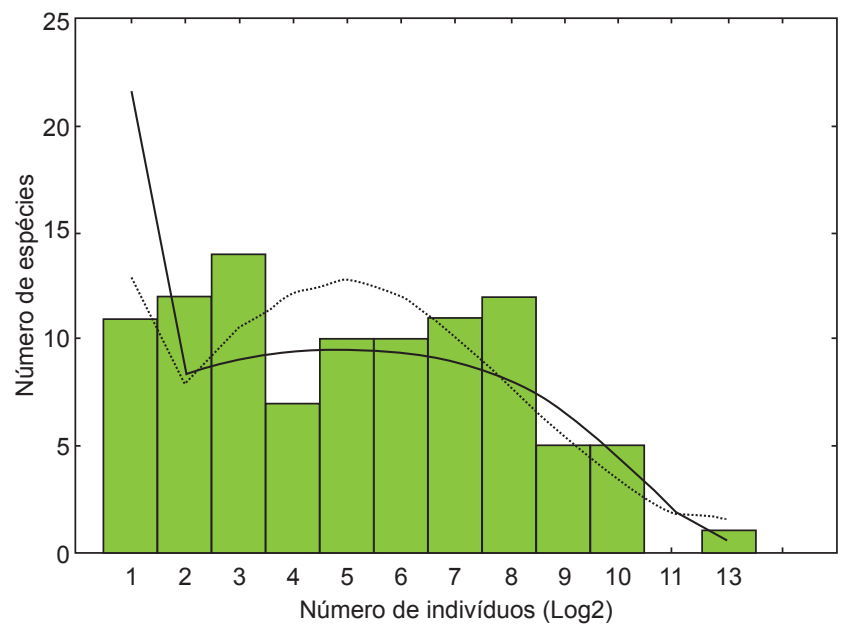

Figura 3. Distribuição das abundâncias de espécies para os meses de junho, setembro e dezembro de 2005, capturadas na zona litoral vegetada de 15 lagoas marginais do rio Cuiabá, Pantanal, Brasil. Os dados são a soma dos três períodos de amostragens junho, setembro e dezembro de 2005. Parâmetros para distribuição lognormal (curva pontilhada) utilizando o método de Pielou (1975), são a média $=1,379$ a variância $=0,719$ e o número de espécie estimada e de 102,6. Para a distribuição log-series (curva contínua) os parâmetros são alfa $=14,441$ e $\mathrm{x}=0,998$. A distribuição $\log$-normal $\left(\mathrm{x}^{2}=12,17 \mathrm{gl}=9\right.$ e $\mathrm{p}>0,20)$ apresentou melhor ajuste do que o log-series $\left(\mathrm{x}^{2}=15,53 \mathrm{gl}=11\right.$ e $\mathrm{p}>0,1$ ).

Figure 3. Fish abundance distribution of the vegetated littoral zone for 15 floodplain lagoons of the Cuiabá River Basin, Pantanal, Brazil. The data are the sum of the three period sampled (June, September, and December of 2005). Lognormal distribution parameters (point line) estimated with Pielou (1975) method are: mean $=1.379$, variance $=0.719$, and species number $=02.6$. For log-series (normal line) the parameters are: $a l f a=14.441$, and $x=0.998$. Both models adjusted the data; lognormal $\left(\mathrm{x}^{2}=12.17\right.$, df $=9$, and $\left.\mathrm{p}>0.20\right)$, and $\log$-series $\left(\mathrm{x}^{2}=15.53, \mathrm{df}=11\right.$, and $\left.\mathrm{p}>0.1\right)$. 
Tabela 1. Distribuição da riqueza de espécies, e abundância individual da zona litoral vegetada de peixes para 15 lagoas da bacia do rio Cuiabá, Pantanal, Brasil.

Table 1. Species richness distribution, and individual abundance of the vegetated littoral zone fishes for 15 floodplain lagoons of the Cuiabá River Basin, Pantanal, Brazil.

\begin{tabular}{|c|c|c|c|c|}
\hline & Junho & Setembro & Dezembro & Total \\
\hline Riqueza & 80 & 74 & 68 & 98 \\
\hline Riqueza acumulada & 80 & 93 & 98 & 98 \\
\hline Riqueza de espécies exclusivas & 10 & 7 & 5 & 22 \\
\hline Abundância total (número) & 5.591 & 5.105 & 2.088 & 12.784 \\
\hline
\end{tabular}

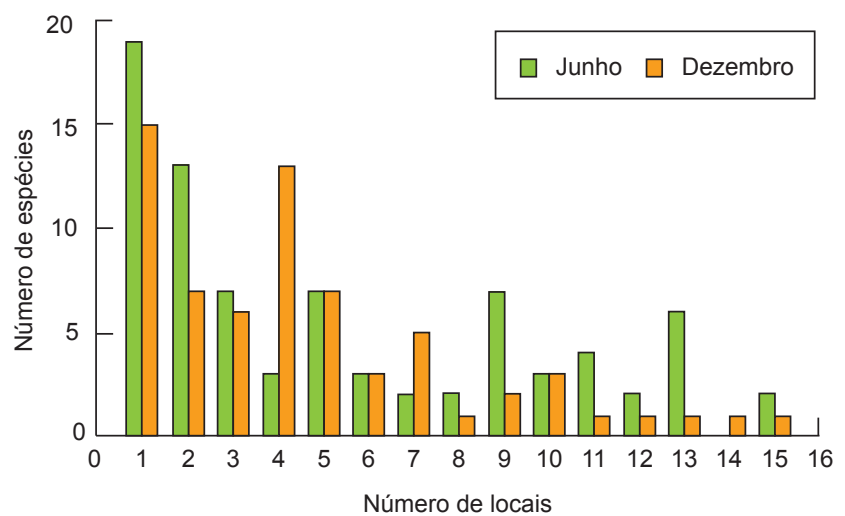

Figura 4. Distribuição de ocorrência das espécies de peixes na zona litoral vegetada da bacia do rio Cuiabá, Pantanal, Brasil. As coletas dos dados iniciaram em junho e encerraram em dezembro.

Figure 4. Distribution of fish species occurrence by lagoon for vegetated littoral zone in the Cuiabá River Basin, Pantanal, Brazil. Data collection started in June and ended in December.

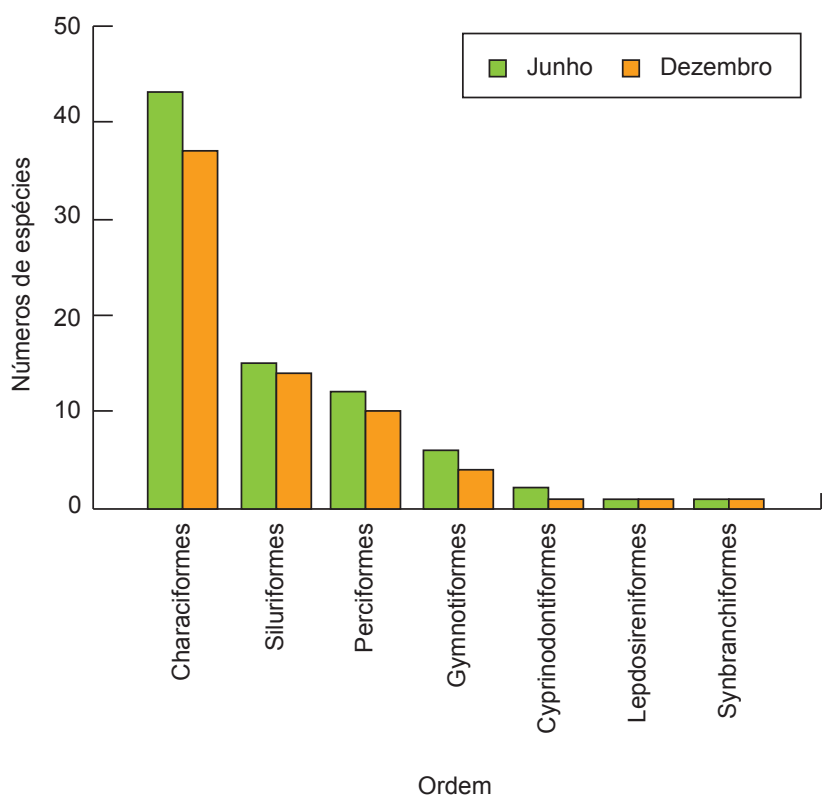

Figura 5. Distribuição da riqueza de espécies agrupadas por ordens taxonômicas na zona litoral vegetada para 15 lagoas da bacia do rio Cuiabá, Pantanal, Brasil. Coletas de dados iniciaram em junho e encerraram em dezembro.

Figure 5. Distribution of fish species richness grouped by taxonomic orders for vegetated littoral zone for 15 floodplain lagoons of the Cuiabá River Basin, Pantanal, Brazil. Data collection started in June and ended in December.

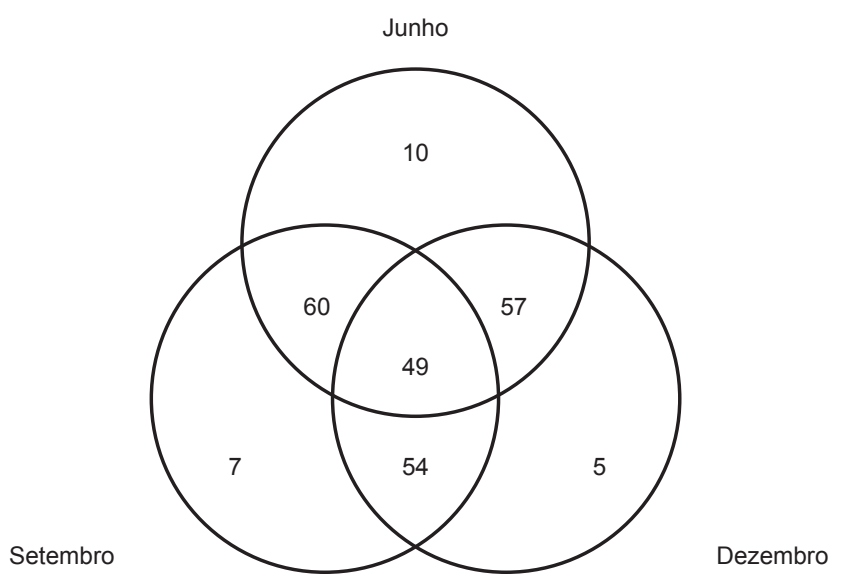

Figura 6. Distribuição da riqueza de espécies de peixes no espaço e no tempo na zona litoral vegetada para 15 lagoas da bacia do rio Cuiabá, Pantanal, Brasil.

Figure 6. Spatio-temporal fish species richness distribution within the vegetated littoral zone for 15 floodplain lagoons in the Cuiabá River Basin, Pantanal, Brazil.

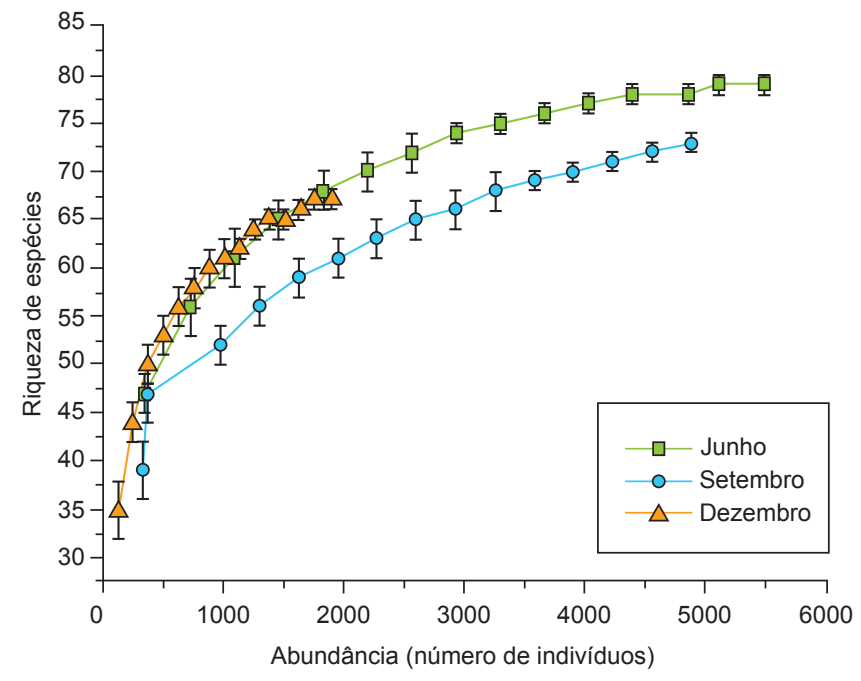

Figura 7. Curvas de rarefação da riqueza de espécies de peixes da zona litoral vegetada para 15 lagoas marginais da bacia do rio Cuiabá, Pantanal, Brasil.

Figure 7. Rarefaction curves of species richness of the vegetated littoral zone fishes for 15 floodplain lagoons in the Cuiabá River Basin, Pantanal, Brazil.

Os estimadores de riqueza de espécies indicaram que distribuir as amostras no tempo foi uma estratégia adequada para reduzir o problema das falsas ausências e melhorar a eficiência da amostragem. Os dados estimados pelo Jackknife de $2^{\text {a }}$ ordem sugerem que no 
mês de junho foram amostradas cerca de $77,7 \%$ do total de espécies esperado (Jackknife $2=103$ espécies; Chao $2=91$, dp =9,99). A eficiência da amostragem foi menor em setembro (70,5\% do total esperado de espécies; Jackknife $2=105$ espécies; Chao $2=100$ espécies, $\mathrm{dp}=16,76)$ e melhorou um pouco em dezembro $(77,3 \%$ do total esperado de espécies; Jackknife $2=88$ espécies; Chao $2=79$, $\mathrm{dp}=8,46$ ). Entretanto, quando se agrupou as amostras, a eficiência da amostragem melhorou em cerca de $12,5 \%$ e foram registradas $84,5 \%$ do total de espécies estimado para a área (Jackknife $2=116$ espécies; Chao $2=105, \mathrm{dp}=5,81$.

\section{Discussão}

A ictiofauna da América do Sul é caracterizada pela dominância de Characiformes sobre Siluriformes (Lowe-McConnell 1999). Essa dominância é evidente tanto em lagoas da porção sul do Pantanal (Súarez et al. 2001, Súarez et al. 2004) quanto na porção norte (presente estudo).

Os resultados deste estudo demonstram que as assembléias estudadas apresentam predominância de espécies com abundâncias moderada e rara, e distribuição espacial restrita. Esse padrão pode resultar da variação entre as espécies na capacidade de dispersão, ou alternativamente, da seleção de habitat e interações populacionais. Possivelmente, as espécies com distribuição espacial mais ampla são abundantes e não apresentam limitação para a dispersão, devido às estratégias adaptativas que as capacitam a explorar ambientes sazonais. Por outro lado, espécies com distribuição espacial restrita devem apresentar limitação de dispersão, sem estratégias adaptativas evidentes para a exploração de ambientes sazonais (Granado-Lorencio et al. 2005)

O padrão de distribuição das espécies entre as lagoas deste estudo, assim como no trabalho de Súarez et al. (2001) - indicando baixa similaridade entre elas - demonstram que, na escala estudada, a diversidade de peixes de lagoas do Pantanal é determinada, principalmente, pela diversidade beta (diversidade entre locais). Assim, para se conservar o conjunto da fauna de peixes de uma das sub-regiões é preferível selecionar um conjunto de lagoas com características variadas (Súarez et al. 2001).

A distribuição de espécies entre as ordens não se alterou ao longo da seca. Esse resultado difere do encontrado por Rodríguez \& Lewis (1997), em lagoas do rio Orinoco, na Venezuela, e por Tejerina-Garro et al. (1998), em lagoas do rio Araguaia, que observaram um aumento na proporção de Siluriformes em relação a Characiformes com o avanço da seca, devido, principalmente, à redução na transparência da água. Essa diferença pode ser decorrente da diferença nos delineamentos amostrais adotados. Diferentemente dos estudos de Rodríguez \& Lewis (1997) e Tejerina-Garro et al. (1998), que amostraram toda a lagoa, nós amostramos peixes apenas na zona litoral vegetada. Além disso, utilizamos apenas um apetrecho de pesca, e com características mais apropriadas à amostragem de peixes de pequeno porte. Alternativamente, nossos resultados podem indicar maior estabilidade nas assembléias da zona litoral vegetada. Nos ambientes pouco profundos das lagoas, as melhores condições de oxigenação da superfície, aliadas à maior disponibilidade tanto de abrigos contra os predadores, quanto de alimentos, fornecido pelas macrófitas (formação de refúgios ecológicos), deve conferir vantagens às formas capazes de melhor explorá-las. Os pequenos Characiformes, como Serrapinnus calliurus, que predominaram nas amostragens parecem se enquadrar nessa categoria (Rodríguez \& Lewis 1997, Veríssimo 1999, Súarez et al. 2004).

Ao longo do período de seca houve redução na riqueza geral e na abundância de indivíduos. As curvas de rarefação indicam que essa redução da riqueza pode estar associada com a menor abundância nos meses de setembro e dezembro. A menor abundância de indivíduos no mês de dezembro, final da estação seca, quando comparada ao mês de junho, início da estação seca, pode estar relacionada a duas razões principais, que atuam em conjunto. A primeira é de ordem demográfica: para a maioria das espécies de peixes da planície de inundação, o recrutamento é um evento discreto no tempo, em geral concentrado nos meses de cheia (Lowe-McConnell 1999), enquanto que a mortalidade é um evento contínuo e, portanto, dominante na fase de seca. A segunda está relacionada à diminuição do nível da água nas lagoas, que intensifica as interações bióticas (competição e predação), excluindo as espécies menos competitivas ou susceptíveis a forte pressão de predação, diminuindo assim a riqueza de espécies no final do período de seca (Hoeinghaus et al. 2003). De fato, Súarez et al. (2004) mostraram que a variação na riqueza é negativamente relacionada com a variação no tempo de isolamento e distância do rio principal, mas positivamente relacionada com a profundidade das lagoas.

Embora Brown (1986) afirme que as espécies que ocorrem no Pantanal têm ampla distribuição regional, apenas metade das espécies foram comuns aos três períodos de coleta e 22 espécies ocorreram em apenas uma lagoa. Isso mostra que as espécies de peixes de pequeno porte que habitam as áreas vegetadas de lagoas marginais do Pantanal, além de não possuírem ampla distribuição espacial, não apresentam ampla distribuição temporal. Provavelmente, isso ocorre devido às estratégias ecológicas distintas que cada espécie possui, e que as permitem manter populações viáveis em um sistema instável como o Pantanal. Conseqüentemente, o conjunto de resultados que encontramos nos leva a sugerir que, na situação de necessidade de delineamento de reservas no Pantanal, a escolha de áreas contendo um grande número de lagoas com tamanhos e formas variadas, é uma opção que permite a conservação da diversidade regional de peixes, pois a maioria das espécies de pequeno porte apresenta distribuição restrita a poucas lagoas.

\section{Agradecimentos}

Ao CNPq/Programa PELD (Sítio 12) e Centro de Pesquisas do Pantanal (CPP)/Ministério da Ciência e Tecnologia (MCT), pelo apoio financeiro. À RPPN SESC Pantanal e seus guarda-parques pelo apoio logístico. Ao Dr. Flávio T. Lima (MZUSP) pelo auxílio na identificação dos peixes. Aos revisores pelas críticas e sugestões. Ao Dr. Pierre Girard (UFMT) pela correção da tradução do abstract. Ao Dr. Roberto de Moraes Lima Silveira (UFMT) pelas correções das traduções das legendas e da tabela.

\section{Referências Bibliográficas}

BEGON, M., TOWNSEND, C.R. \& HARPER. J.L. 2006. Ecology: from individuals to ecosystems. 4 ed. Blackwell Publishing, Oxford.

BRITSKI, H.A., SILIMON, K.Z.S. \& LOPES, B.S. 1999. Peixes do Pantanal: manual de identificação. EMBRAPA, Brasília.

BROWN JUNIOR, K.S. 1986. Zoogeografia da região do Pantanal Matogrossense. In Simpósio sobre recursos naturais e socioeconômicos do Pantanal, Embrapa-Corumbá, MS, p. 137-178.

CHALCRAFT, D.R, WILLIAMS, J.W., SMITH, M.D. \& WILLIG, M.R. 2004. Scale dependence in the species-richness-productivity relationship: the role of species turnover. Ecology 85(10):2701-2708.

COLWELL, R.K. 2005. EstimateS: statistical estimation of species richness and shared species from samples. Version 7.5. Persistent URL < purl. oclc.org/estimates>.

DeVRIES, P.J., MURRAY, D. \& LAND, R. 1997. Species diversity in vertical, horizontal, and temporal dimensions of a fruit-feeding butterfly community in an Ecuadorian rainforest. Biol. J. Linn. Soc. 62:343-364.

GOTELLI, N.J. \& GRAVES, G.R. 1996. Null models in ecology. Smithsonian Institution Press, Washington and London. 
GRANADO-LORENCIO, C., LIMA, R.M. A. \& LOBÓN-CERVIÁ, J. 2005. Abundance-distribution relationships in fish assembly of the Amazonas floodplain lakes. Ecography 28:515-520.

HAMILTON, S.K., SIPPEL, S.L. \& MELACK, J.M. 1996. Inundation patterns in the Pantanal wetland of South American determined from passive remote sensing. Arch. Hydrobiol. 137:1-23.

HINCH, S.G. \& COLLINS, N. 1991. Relative abundance of littoral zone fishes: biotic interactions, abiotic factors, and postglacial colonization. Ecology 72(4):1314-1324.

HOEINGHAUS, D.J., LAYMAN, C.A.,ARRINGTON,D.A.\& WINEMILLER, K.O. 2003. Spatiotemporal variation in fish assemblage structure in tropical floodplain creeks. Environ. Biol. Fish. 67(4):379-387.

LOWE-McCONNELL, R.H. 1999. Estudos ecológicos de comunidades de peixes tropicais. Edusp, São Paulo.

LUGENDO, B.R, A. de GROENE, I. CORNELISSEN, A. PRONKER, I. NAGELKERKEN, G. VAN DER VELDE, Y.D. MGAYA. 2007. Spatial and temporal variation in fish community of a marine embayment in Zanzibar, Tanzania. Hydrobiol. 586(1):1-16.

MAGURRAN, A.E 1988a. BIODAP. Ecological diversity and its measurement. Resource Conservation Fundy National Park, Alma.

MAGURRAN, A.E. 1988. Ecological diversity and its measurement. Princeton University Press, Princeton.

MAGURRAN, A.E. 2004. Measuring biological diversity. Blackwell Publishing, Oxford.
MIRANDA, J.C. \& MAZZONI, R. 2003. Composição da ictiofauna de três riachos do alto rio Tocantins-GO. Biota Neotrop. (1):http:/www. biotaneotropica.org.br/v3n1/pt/abstract?article+BN00603012003.

PIELOU, F.C. 1975. Ecological diversity. Wiley, New York.

REIS, R.E., KULLANDER, O. \& FERRARIS JR, C.J. 2003. Check list of the freshwater fishes of South and Central America. EDIPUCRS, Porto Alegre.

RODRÍGUEZ, M.A. \& LEWIS, M.L. 1997. Structure of fish assemblages along environmental gradients in floodplain lakes of the Orinoco river. Ecol. Monogr. 67(1):109-128.

SANDERS, H.L. 1968. Marine benthic diversity: a comparative study. Am. Nat. 102:243-282.

SÚAREZ, Y.R., PETRERE-JÚNIOR, M. \& CATELLA, A.C. 2001. Factors determining the structure of fish communities in Pantanal lagoons (MS, Brazil). Fisheries Manag. Ecol. 8:173-186.

SÚAREZ, Y.R., PETRERE-JÚNIOR, M. \& CATELLA, A.C. 2004. Factors regulating diversity and abundance of fish communities in Pantanal lagoons, Brazil. Fisheries Manag. Ecol. 11:45-50.

TEJERINA-GARRO, F.L., FORTIN, R. \& RODRIGUEZ, M.A. 1998. Fish community structure in relation to enviromental variation in floodplain lakes of the Araguaia River, Amazon Basin. Environ. Biol. Fish. 51(4):399-410.

VERÍSSIMO, S. 1999. Influência do regime hidrológico sobre a ictiocenose de três lagoas da planície aluvial do alto rio Paraná. Tese de Doutorado. UFSCar, São Carlos.

WEBSTER, M.S. 2003. Temporal density dependence and population regulation in a marine fish. Ecology 84(3):623-628. 